\title{
Rapid Diagnosis of Malaria by Antigen Detection
}

\author{
Mejbah Uddin Ahmed, ${ }^{1}$ Md. Akram Hossain,,2 AKM Shamsuzzaman, ${ }^{2}$ Md. Murshed Alam, ${ }^{3}$ Abul Hossain Khan, ${ }^{4}$ Asma Azad \\ Sumona, ${ }^{2}$ Ahmed Nawsher Alam, ${ }^{5}$ Salma Ahmed, ${ }^{2}$ Mahmuda Siddiqua 6 \\ ${ }^{1}$ Department of Microbiology, Enam Medical College, Savar, Dhaka; 2Department of Microbiology, Mymensingh Medical College, \\ Mymensingh; ${ }^{3}$ Department of Microbiology, Community based Medical College, Mymensingh; ${ }^{4}$ Department of Microbiology, \\ Kwaza Younus Ali Medical College, Enayetpur, Sirajganj; 5 Department of Microbiology, Institute of Public Health, Mohakhali, \\ Dhaka; 'Department of Microbiology, Ibn Sina Medical College, Dhaka
}

\begin{abstract}
The study was conducted to evaluate the sensitivity and specificity of Immunochromatographic test (ICT) for antigen, using microscopy as the "gold standard" method for diagnosis of malaria. A total of 98 clinically suspected malaria patients and another 30 age and sex-matched healthy controls were included in this study. Thick and thin films were also prepared and examined under microscope as well as Immunochromatographic test (ICT) was performed for malaria antigen. Sensitivity and specificity of ICT for antigen were $93.22 \%$ and $\mathbf{9 4 . 8 7 \%}$ respectively.
\end{abstract}

Keywords: Detection of malaria antigen, Immunochromatographic test

\section{Introduction}

Malaria, caused by different species of the genus Plasmodium infect red blood cells, is one of the many febrile illnesses that cause substantial morbidity and mortality throughout the world. In the recent years, malaria situation has been worsening in Bangladesh. ${ }^{1,2}$

A prompt and accurate diagnosis is the key to effective disease management in malaria. ${ }^{3}$ Several laboratory procedures such as different types of microscopy, immunological methods for antigen and antibody detection, polymerase chain reaction, species-specific DNA probe and ribosomal RNA probe have been developed. ${ }^{4}$ Among the methods, fluorescence microscopy and molecular techniques are highly sensitive $(99.5 \%)$ and specific (100\%) but needs

\section{Correspondence:}

Dr. Mesbah Uddin Ahmed

Assistant Professor

Department of Microbiology

Enam Medical College, Savar, Dhaka

E-mail : mejbah@ssadikk.com considerable amount of practice with costly equipments..$^{4,5}$

The most widely used approach to confirmatory diagnosis of malaria is demonstration of Plasmodium species by microscopic examination of thick and thin films. This is regarded as the "gold standard" method and is relatively simple with low costs. ${ }^{6-8}$ But it is time consuming, laborious and sensitivity is questionable at low level of parasitemia. Its interpretation is troublesome in case of mixed infection. ${ }^{9-11}$

One of the newly developed technologies for rapid diagnosis of malaria is the detection of antigen by immunochromatographic test (ICT) method. In situations, where reliable microscopy may not be available, the ICT method may be very effective. Malaria antigens currently targeted by rapid diagnostic tests (RDT) are Histidine-rich proteins 2 (HRP-2), Plasmodium lactate dehydrogenase (pLDH) and Plasmodium aldolase. ${ }^{3}$ After successful treatment, $\mathrm{pLDH}$ is cleared rapidly from the body but pfHRP2 may persist up to 7 to 10 days. Thus detection of $\mathrm{pLDH}$ 
antigen may help to monitor the effectiveness of the treatment. ${ }^{12}$

Though methods for antigen detection of malaria are available commercially, but no study to evaluate those kits were conducted in the local institute as well as nearly endemic areas. For this reason, the study was carried out to see the diagnostic efficacy of immunochromatographic test for the detection of malarial antigen.

\section{Methods}

This case-control study was carried out in the Department of Microbiology, Mymensingh Medical College for a period of one year from July, 2005 to June, 2006. A total of 98 cases were selected from three healthcare facilities of Mymensingh, namely Haluaghat Thana Health Complex, Mymensingh Medical College Hospital, and Community Based Medical College Hospital. Cases were selected on the basis of fever, associated with chills and rigor, sweating, splenomegaly, hepatomegaly, headache, fatigue and abdominal discomfort. Another 30, age and sex-matched, healthy persons were included as controls from the same areas. All relevant history, clinical findings and laboratory records of every case and control was systematically recorded in a pre-designed data sheet and subsequently analyzed by computer programme SPSS version 12.0.

Capillary blood from the tip of the finger for thick and thin film, and intravenous blood for immunochromatographic test was collected aseptically. The slides of thick and thin films were labeled and stained with Giemsa stain. ${ }^{13}$ Antigen was detected by immunochromatographic (ICT) method with SD Bioline malaria antigen test kit.

\section{Detection of Antigen}

The SD Bioline malaria antigen test kit contains a membrane strip, which is pre-coated with two polyclonal antibodies as two separate lines across a test strip. In one line, polyclonal antibodies specific to lactate dehydrogenase of $P$. falciparum and in other line plolyclonal antibodies to lactate dehydrogenase of common to all four species ( $P$. falciparum, $P$. vivax, $P$. malariae and $P$. ovale) were used.

Conjugate and washing wells were inserted into microplate frame. One drop of assay buffer into the conjugate well and 4 drops into the washing well were dispensed. An amount of 20 $\mu \mathrm{l}$ of freshly collected blood was added into the conjugate well, and mixed gently. The dipstick was then placed vertically into the conjugate well including specimen for 5 minutes. After that dipstick was transferred from the conjugate well to the washing well, until the dipstick was cleared of blood, was removed from the washing well and result was taken.

\section{Results}

Out of 98 clinically suspected cases, 59 (60.20\%) were positive by microscopic examination of peripheral blood film and $57(58.16 \%)$ were positive by ICT for antigen. (Table I)

Sensitivity and specificity of ICT for antigen when compared with microscopic examination of peripheral blood film were calculated. The sensitivity and specificity were $93.22 \%$ and $94.87 \%$ respectively. (Table II)

Table I: Rate of detection of malaria by different methods

\begin{tabular}{lll}
\hline Tests & \multicolumn{2}{c}{ Results } \\
& Positive & Negative \\
\hline Peripheral blood film $(\mathrm{n}=98)$ & $59(60.20 \%)$ & $39(39.80 \%)$ \\
ICT for malarial antigen $(\mathrm{n}=98)$ & $57(58.16 \%)$ & $41(41.84 \%)$ \\
\hline
\end{tabular}

Table II: Sensitivity and specificity of ICT for antigen when compared with microscopic examination of peripheral blood film

\begin{tabular}{lllll}
\hline Test result & $\begin{array}{l}\text { Disease } \\
\text { positive } \\
(\mathrm{MP}+\mathrm{ve})\end{array}$ & $\begin{array}{l}\text { Disease } \\
\text { negative } \\
(\mathrm{MP}-\mathrm{ve})\end{array}$ & Sensitivity & Specificity \\
\hline ICT antigen +ve & 55 & 02 & & \\
ICT antigen -ve & 04 & 37 & $93.22 \%$ & $94.87 \%$ \\
Total & 59 & 39 & & \\
\hline
\end{tabular}

\section{Discussion}

Detection of antigens by Immunochromatographic test method has been introduced very recently and is simple, rapid, reliable and therefore, suitable. ${ }^{10}$

In the present study, among the clinically suspected cases, 59 $(60.20 \%)$ were positive by microscopic examination of peripheral blood film, and 57 (58.16\%) were positive by ICT 
for antigen. In a study by Khan et al in 2004 from Pakistan found $45.5 \%$ positive by microscopic examination of peripheral blood film and $43.2 \%$ positive by ICT for antigen. ${ }^{14}$ In another study by Palmer et al in 1998 from United States of America found $48 \%$ positive by microscopy and $45 \%$ positive by ICT for antigen. ${ }^{15}$ From the above findings, it is observed that some results are close to and some remarkably differ from the present study. The difference may be due to the fact that studies were conducted at different geographical areas and the disease prevalence differs from region to region.

In the present study, sensitivity and specificity of ICT for Plasmodial antigen detection were $93.22 \%$ and $94.87 \%$ respectively, when compared with microscopic results of peripheral blood film. In a study from Australia by Playford and Walker in 2002 showed sensitivity of $85 \%$ and specificity of $96 \%$ when compared with microscopic examination of peripheral blood film. ${ }^{16}$ In another study from Munich, Germany by Jelinek et al in 1999 showed sensitivity of $92.5 \%$ and a specificity of $98.3 \% .{ }^{17}$ Regarding sensitivity and specificity, these results are nearly similar to the present study.

Though expensive, rapid tests are simple to perform and diagnostic efficacy is satisfactory. So, this method can be used selectively.

\section{References}

1. Keiser J, Utziner J, DE Castro CM, Thomas A, Smith TA, Tanner M, et al. Urbanization in Sub-Saharan Africa and implication for malaria control. Am J Trop Med Hyg 2004; 71: 118-127.

2. International Center for Diarrhoeal Diseases and Research, Bangladesh. New strategies for treating falciparum malaria in Bangladesh. Health Sc Bull 2006; 4: 1-6.

3. Kurshid M, Harani MS, Beg MA, Khaleeq L, Adil SN, Kakepoto GN. Role of ICT Malaria Immunochromatographic Test for Rapid diagnosis of Malaria. J Pakistan Med Assoc 2006; 56: 167.

4. Nandwani S, Mathur M, Rawat S. Evaluation of the polymerase chain reaction analysis for diagnosis of falciparum malaria in Delhi, India. Indian J Med Microbiol 2005; 23: 176-178.

5. Farcas GA, Zhong KJY, Mazzulli T, Kain KC. Evaluation of the RealArt Malaria LC Real-Time PCR Assay for Malaria Diagnosis. J Clin Microbiol 2004; 42: 636-638.

6. Saeed AA, Al Rasheed AM, Al Nasser I, Al Onaizi M, Al
Kahtani S. Malaria Screening of Blood Donors in Saudi Arabia. Ann Saudi Med 2002; 22: 329-332.

7. Kong HH, Chung D-II. Comparison of acridine orange and Giemsa stains for malaria diagnosis. Korean J Parasitol 1995; 33: 391-394.

8. Mankhambo L, Kanjala M, Rudman S, Lema VM, Rogerson SJ. Evaluation of the Optimal Rapid Antigen Test and Speciesspecific PCR to Detect Placental Plasmodium falciparum Infection at Delivery. J Clin Microbiol 2002; 40: 155-158.

9. Hamer DH, Ndhlovu M, Zurovac D, Fox M, Yeboah-Antwi K, Chanda $\mathrm{P}$, et al. Improved Diagnostic Testing and Malaria Treatment Practices in Zambia. J Am Med Assoc 2007; 297: 2227-2231.

10. Joshi HH. Monoclonal Antibody Based ELISA: An Effective Diagnostic Tool For The Diagnosis of Falciparum Malaria. J Nepal Med Assoc 2005; 44: 79-83.

11. Moody A. Rapid Diagnostic Tests for Malaria parasites. Clin Microbiol Rev 2002; 15: 66-78.

12. Iqbal J, Khalid N, Hira PR. Comparison of Two Commercial Assays with Expert Microscopy for confirmation of symptomatically Diagnosed Malaria. J Clin Microbiol 2002; 40: 4675-4678

13. Cheesbrough M. Examination of blood for malarial parasites. In: District Laboratory practice in Tropical Countries, Part-1. United Kingdom: Cambridge University press, 1999: pp. 239258.

14. Khan SA, Anwar M, Hussain S, Qureshi AH, Ahmad M, Afzal S. Comparison of Optimal Malarial Test with Light Microscopy for the diagnosis of Malaria. J Pakistan Med Assoc 2004; 54: 404-408.

15. Palmer CJ, Lindo JF, Klaskala WI, Quesada JA, Kaminsky R, Baum MK, et al. Evaluation of the Optimal Test for Rapid Diagnosis of Plasmodium vivax and Plasmodium falciparum Malaria. J Clin Microbiol 1998; 36: 203-206.

16. Playford EG, Walker J. Evaluation of the ICT malaria p.f/p.v and the optimal rapid diagnostic tests for malaria in febrile returned travelers. J Clin Microbiol 2002; 40: 4166-4171.

17. Jelinek T, Grobusch MP, Schwenke S, Steidl S, Sonnenburg F, Nothdurft HD, et al. Sensitivity and specificity of dipstick tests for rapid diagnosis of malaria in nonimmune travelers. J Clin Microbiol 1999; 37: 721-723.

[ Conflict of Interest: none declared] 